\title{
Outcomes From a Patient-Centered, Interprofessional, Palliative Consult Team in Oncology
}

\author{
Andrea Feldstain, PhD ${ }^{\mathrm{a}, \mathrm{b}}$; Barry D. Bultz, PhD ${ }^{\mathrm{a}, \mathrm{b}}$; Janet de Groot, MD, FRCPC, MSc ${ }^{\mathrm{a}, \mathrm{c}}$; \\ Amane Abdul-Razzak, MD, CCFP, MSc ${ }^{\mathrm{d}, \mathrm{e}}$; Leonie Herx, MD, PhD, CCFP (PC) ${ }^{\mathrm{d}, \mathrm{e}}$; \\ Lyle Galloway, MD, CCFP (PC) ${ }^{\mathrm{d}, \mathrm{e}}$; Srini Chary, MBBS, MRCS, LRCP, CCFP (PC), DA, FRCSEd ${ }^{\mathrm{d}, \mathrm{e}}$; \\ and Aynharan Sinnarajah, MD, MPH, CCFP (PC) d,e
}

\begin{abstract}
Background: Palliative care aims to improve suffering and quality of life for patients with life-limiting disease. This study evaluated an interdisciplinary palliative consultation team for outpatients with advanced cancer at the Tom Baker Cancer Centre. This team traditionally offered palliative medicine and recently integrated a specialized psychosocial clinician. Historic patient-reported clinical outcomes were reviewed. There were no a priori hypotheses. Methods: A total of 180 chart reviews were performed in 8 sample months in 2015 and 2016 ; 114 patients were included. All patients were referred for management of complex cancer symptomatology by oncology or palliative care clinicians. Patients attended initial interviews in person; palliative medicine follow-ups were largely performed by telephone, and psychosocial appointments were conducted in person for those who were interested and had psychosocial concerns. Chart review included collection of demographics, medical information, and screening for distress measures at referral, initial consult, and discharge. Results: A total of $51 \%$ of the patient sample were men, $81 \%$ were living with a partner, and $87 \%$ had an advanced cancer diagnosis. Patients were grouped based on high, moderate, or low scores for 5 symptoms (pain, fatigue, depression, anxiety, and well-being). High scores on all 5 symptoms decreased from referral to discharge. Pain and anxiety decreased in the moderate group. All 5 low scores increased significantly. Sleep, frustration/anger, sense of burdening others, and sensitivity to cold were less frequently endorsed by discharge. Conclusions: Patients who completed this interdisciplinary palliative consult service appeared to experience a reduction in their most severe symptoms. Visits to patients during existing appointments or having them attend a half-day clinic appears to have reached those referred. With interdisciplinary integration, clinicians are able to collaborate to address patient care needs. Considerations include how to further integrate palliative and psychosocial care to achieve additional benefits and ongoing monitoring of changes in symptom burden.
\end{abstract}

J Natl Compr Canc Netw 2018;16(6):719-726 doi: 10.6004/jncen.2018.7014

\section{Background}

Palliative care is an effective interprofessional approach empirically and internationally supported to reduce suffering through improvement of symptoms and quality of life (QoL). ${ }^{1-3}$ Since its inception in $1967,{ }^{4}$ palliative care has recognized that suffering includes physical, psy-

aDepartment of Psychosocial and Rehabilitation Oncology, Tom Baker Cancer Centre, Alberta Health Services; 'Division of Psychosocial Oncology, 'Psychiatry and Oncology, and dDivision of Palliative Medicine, Cumming School of Medicine, University of Calgary; and ePalliative/End-of-Life Care, Calgary Zone, Alberta Health Services, Calgary, Alberta, Canada.

Submitted October 14, 2017; accepted for publication February 6, 2018.

The authors have disclosed that they have no financial interests, arrangements, affiliations, or commercial interests with the manufacturers of any products discussed in this article or their competitors.

Dr. Feldstain was supported by a post-doctoral fellowship from the Alberta chological, social, and existential causes and aims to address suffering as part of whole patient care.

The Palliative and End of Life Care program in Calgary, Canada, provides services through multiple teams. The Complex Cancer Management Service (CCMS)

Cancer Foundation, Cancer Control Alberta, the Tom Baker Cancer Centre, and the Daniel Family Foundation.

Author contributions: Ethics preparation and submission: All authors. Patient assessment and intervention: Galloway, Chary, Abdul-Razzak, Feldstain. Data collection and analysis: Feldstain. Manuscript preparation: All authors.

Correspondence: Andrea Feldstain, PhD, Department of Psychosocial and Rehabilitation Oncology, Tom Baker Cancer Centre, Alberta Health Services, 2202 2nd Street SW, Calgary, Alberta, Canada, T2C 3C1.

Email: andrea.feldstain@ahs.ca 
outpatient consultation team at Tom Baker Cancer Centre (TBCC) has traditionally included physicians and a nurse practitioner (palliative consultants), a registered nurse (triage), and pharmacists. Psychosocial intervention was obtained by clinicianor self-referral to the TBCC Department of Psychosocial and Rehabilitation Oncology (PSRO).

The TBCC PSRO is an outpatient interprofessional team that offers specialized psychosocial services for individuals, couples, families, and support persons affected by cancer. Psychosocial clinicians include clinical psychologists, social workers, and psychiatrists. The PSRO functions in a tumor-group model, with one clinician dedicated to 1 or 2 particular tumor types, specializing in the particular psychosocial needs of that patient population. A benefit of this model is that communication, collaboration, research, and consultation among the tumor-group's medical and psychosocial clinicians is bolstered, potentially improving the quality and continuity of patient care. ${ }^{5}$ General referrals to the PSRO are also possible for tumor groups without a dedicated psychosocial clinician, although this lacks the element of interprofessional integration.

The CCMS did not have a dedicated psychosocial clinician until 2015, when training grants allowed TBCC to pilot this integration for 2 years. In contrast to the previous method of making general referrals to the PSRO, the new dedicated psychosocial clinician worked within the CCMS team. She was available to join initial patient visits with other CCMS team members. She also worked closely with the inpatient acute palliative care unit on site, allowing clinician continuity between inpatient and outpatient settings. Integration in these ways is of particular importance in CCMS patient populations who demonstrate a "complex" symptom burden, often influenced by a number of biopsychosocial concerns. ${ }^{6-8}$ It allows for better coordinated interprofessional approaches and treatment planning.

The current goal is to examine clinically reported symptoms by patients in the CCMS program from admission to discharge. Identifying how the CCMS is addressing concerns and identifying unmet needs can help shape the evolution of this service and inform program development. This project was exploratory and had no a priori hypotheses.

\section{Methods}

\section{Participants}

Eligible patients had their first CCMS appointment in January, April, July, or October of either 2015 or 2016 (ie, sampled quarterly over 2 years). Referrals were from oncology or palliative care clinicians for patients experiencing complex cancer symptoms, including physical symptoms (pain, nausea) or psychosocial-spiritual concerns (depression, anxiety, illness adjustment). For pain referrals, a prerequisite to referral was a trial of one strong opioid for nociceptive pain or one first-line agent for neuropathic pain. Patients may also have been referred for reasons unrelated to immediate symptom management needs, such as conversations about goals of care, introduction to palliative services, assistance with conflict resolution or decision-making, or eligibility assessment for tertiary care admission. Patients who did not have any immediate symptom management needs would have been excluded from the current sample, although they would have still received clinical care.

\section{Procedure}

Patient referrals were received by the CCMS triage nurse, who conducted a chart review and contacted eligible patients to schedule their initial appointment. There were 2 ways to access the CCMS. First, patients who needed to be seen urgently could be visited by the CCMS during another TBCC appointment or could be scheduled for an appointment with the CCMS within the following week; this part of the team includes a pharmacist and either a physician or nurse practitioner. The psychosocial clinician may have been asked to attend if referrals indicated psychosocial concerns. Second, if patients were able to wait 2 to 3 weeks and/or did not have upcoming appointments at TBCC, they could schedule an appointment at the CCMS weekly half-day clinic. At the clinic, they met with a physician, pharmacist, and psychosocial clinician. All patients had completed screening for distress (SFD) measures, ${ }^{9}$ discussed in the following section, when meeting any of their healthcare teams as part of routine care at TBCC.

CCMS medicine follow-up was performed via telephone by one of the team's pharmacists. Notes on progress, medication changes, concerns, or discharges were recorded in the patient's file. Pharma- 
Interdisciplinary Palliative Consult

cists could request that patients return to see the team in clinic if necessary. An official discharge from the CCMS was not formalized. Chart notes may have included reasons for ceasing follow-ups, such as patients reporting satisfaction with ameliorated symptom burden, transfer to another palliative care team (eg, inpatient palliative care, palliative home care, hospice care), or death. Psychosocial follow-up was arranged at initial meeting if patients were interested and had psychosocial needs. If the psychosocial clinician was not present, team members could contact her directly to follow-up with the patient or could provide her contact information for patient self-referral. Psychosocial intervention continued to be offered as long as psychosocial need was present. Therefore, "discharge" herein refers to discharge from the palliative medicine component; psychosocial care may have been ongoing.

Chart review was performed to collect SFD data at time of CCMS referral (referral "TO"), initial CCMS appointment (initial consult "T1"), and when completing care with the CCMS (discharge "T2"). Measure T0 was the most recent SFD immediately preceding T1. Two difficulties in data collection occurred: (1) T0 usually occurred within a few weeks before T1 and mostly corresponded with referral, although for some patients TO measures may have been $>1$ month prior to T1 and may not have been at time of referral, and (2) with no formal discharge, T2 may have been estimated around the time that CCMS follow-up notes ceased. This study was approved by the local research ethics board (HREBA.CC-16-0262).

\section{Measures}

The minimum SFD toolkit ${ }^{10}$ includes 2 self-report measures: the Edmonton Symptom Assessment System (ESAS) and the Canadian Problem Checklist (CPC). These forms assess domains of suffering (eg, emotional, physical, social, spiritual) to identify current concerns and potential need for follow-up or referral. The local version used in this study can be found on the Alberta Health Services website. ${ }^{11}$

Edmonton Symptom Assessment System: The ESAS $^{12}$ is a self-report questionnaire that asks patients to rate the severity of their current suffering regarding 9 common symptoms (pain, fatigue, tiredness, nausea, appetite, shortness of breath, depression, anxiety, well-being). Patients are asked to rate each symptom on a visual analog scale ranging from 0 (no symptom) to 10 (worst possible symptom). Use of the ESAS in palliative care has been well validat$\mathrm{ed}^{12-15}$; however, it is considered a screening measure and not intended or appropriate for diagnostic purposes. Results in this study focus on outcomes regarding pain, fatigue, depression, anxiety, and well-being.

Canadian Problem Checklist: The CPC is a checklist of the most commonly reported problems among patients. It was developed by the SFD Toolkit Working Group of the Canadian Partnership Against Cancer to enhance the information gathered with the ESAS. ${ }^{10}$ The version of this checklist used at TBCC includes common problems divided into 7 domains of functioning (physical, emotional, social/spiritual, mobility, informational, nutritional, practical). ${ }^{11}$

\section{Chart Review}

Demographic and medical information, including age, sex, marital status, and cancer site and stage, were obtained by TBCC chart review.

\section{Data Analysis}

Multiple imputation was performed for missing data using NORM. ${ }^{16}$ Variables included for estimation were age, sex, marital status, social support present at T1, and SFD variables at T0, T1, and T2.

Statistical procedures were performed using SPSS version 19.0 (IBM Corporation). Descriptive statistics were used to qualify the sample. Repeated measures analyses were used to assess change over time in ESAS symptoms, and the between-group variable was severity rating (low, 0-3; moderate, 4-6; or high, 7-10) at T0. Changes were considered statistically significant at $P<.05$. Changes in scores were considered clinically significant if change was $\geq 0.5$ standard deviations ${ }^{17}$ and was $\geq 1$ unit. ${ }^{18}$ Finally, Cochrane's $Q$ test was used to assess significant changes in frequency of endorsed items on the CPC between $\mathrm{T} 1$ and $\mathrm{T} 2$.

\section{Results}

A total of 180 patients were scheduled for an initial appointment in the 8 sample months. Of these, 168 completed T1 (93.3\%), 115 completed T2, and 114 were considered in the final sample $(67.9 \%$ of $\mathrm{T} 1)$ (Figure 1). Missing values were imputed for $0.4 \%$ of total data set. 


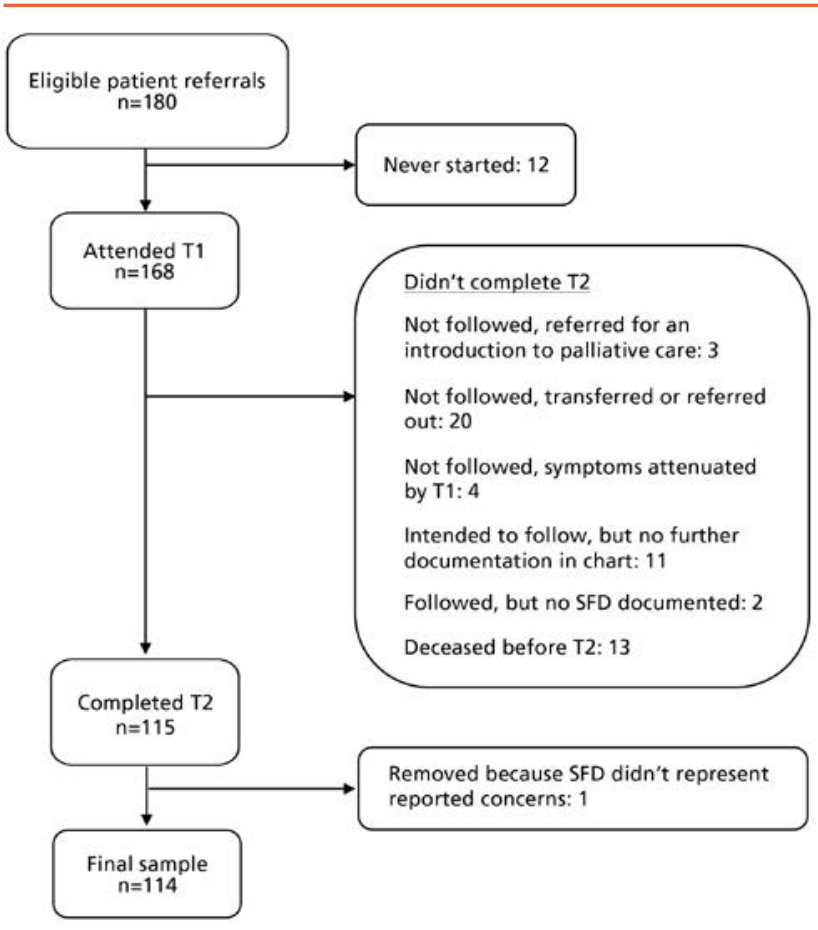

Figure 1. Flowchart of participation.

Abbreviations: SFD, screening for distress; T1, initial consult with the Complex Cancer Management Service; T2, discharge.

Mean time from T0 to T1 was 3.2 weeks (SD, 4.5; median, 2.0; range, 1-36 weeks). Mean duration of admission from $\mathrm{T} 1$ to $\mathrm{T} 2$ was 3.4 months (SD, 3.0; median, 3.0; range, 1 week to 4 . The sample was $50.9 \%$ male and mean age was 59.8 years (SD, 12.8; median, 61.0; range, 25.0-84.0 years). Most were married $(71.1 \%)$, and $64.9 \%$ had a loved one present at $\mathrm{T} 1$. The most represented tumor sites were lung (21.1\%), head and neck (14.0\%), and upper gastrointestinal (14.0\%); 80.7\% had stage III or IV disease. Participants in $2015(\mathrm{n}=52)$ had a mean survival of 8.5 months (SD, 6.3; median, 6.0) and $78.8 \%$ lived $\geq 6$ months. Participants in $2016(n=62)$ had a mean survival of 5.2 months (SD, 3.9; median, 5.0) and $59.6 \%$ lived $\geq 6$ months (Table 1 ). TBCC psychosocial resources (psychology, social work, psychiatry) were used by $49.1 \%$ of the sample during their admission with the CCMS. Analysis of psychosocial outcomes is being prepared as a separate publication.

ESAS items of interest were analyzed using repeated measures analyses. Three potential confounders were examined separately as between-subject variables: (1) which CCMS team was seen (interval between T0-T1 was $\geq 1$ or $<1$ week); (2) whether the interval between T0-T1 was $\geq 1$ or $<1$ month;

\begin{tabular}{|c|c|c|}
\hline & $\mathrm{n}$ & $\%$ \\
\hline \multicolumn{3}{|l|}{ Age, y } \\
\hline Mean [SD] & \multicolumn{2}{|c|}{$59.8[12.8]$} \\
\hline Range & \multicolumn{2}{|c|}{$25.0-84.0$} \\
\hline \multicolumn{3}{|l|}{ Sex } \\
\hline Male & 58 & 50.9 \\
\hline Female & 56 & 49.1 \\
\hline \multicolumn{3}{|l|}{ Marital status } \\
\hline Married/Cohabitating & 81 & 71.1 \\
\hline Divorced & 12 & 10.5 \\
\hline Single & 8 & 7.0 \\
\hline Widow & 3 & 2.6 \\
\hline No spouse documented & 10 & 8.8 \\
\hline \multicolumn{3}{|c|}{ Cancer stage at initial CCMS visit } \\
\hline 1 & 8 & 7.0 \\
\hline$\underline{\text { II }}$ & 13 & 11.4 \\
\hline III & 11 & 9.6 \\
\hline IV & 81 & 71.1 \\
\hline No active cancer & 1 & 0.9 \\
\hline \multicolumn{3}{|l|}{ Primary tumor site } \\
\hline Lung & 24 & 21.1 \\
\hline Head \& neck & 16 & 14.0 \\
\hline Upper gastrointestinal & 16 & 14.0 \\
\hline Hematologic & 13 & 11.4 \\
\hline Colorectal & 13 & 11.4 \\
\hline Breast & 11 & 9.6 \\
\hline Genitourinary & 7 & 7.0 \\
\hline Gynecologic & 8 & 6.1 \\
\hline Central nervous system & 2 & 1.8 \\
\hline Melanoma & 1 & 0.9 \\
\hline Multiple primaries & 1 & 0.9 \\
\hline Unknown primary & 2 & 1.8 \\
\hline \multicolumn{3}{|c|}{ Survival after discharge (T2), mo } \\
\hline \multicolumn{3}{|l|}{2015} \\
\hline$<6$ & 11 & 21.2 \\
\hline 6 to $<12$ & 13 & 25.0 \\
\hline$\geq 12$ & 28 & 53.8 \\
\hline \multicolumn{3}{|l|}{2016} \\
\hline$<6$ & 25 & 40.3 \\
\hline 6 to $<12$ & 11 & 17.7 \\
\hline$\geq 12$ & 26 & 41.9 \\
\hline
\end{tabular}

Abbreviations: CCMS, Complex Cancer Management Service; T2, discharge.

and (3) whether survival time after T2 was $\geq 1$ or $<1$ month. This third potential confounder was included because symptom burden has been found to markedly worsen in the last month of life for this population, ${ }^{19,20}$ and therefore may affect our findings. None were significant in any of the analyses and were removed.

We observed a phenomenon resembling regression to the mean ${ }^{21}$ between T1 and T2: high and low scores appeared more extreme at $\mathrm{T} 1$ and regressed toward the mean by T2. To control for this, we focused on linear trends across the 3 time points for these 
analyses. Table 2 and Figure 2 provide additional detail on the following symptom-related information.

\section{Edmonton Symptom Assessment System}

Pain: The linear trend was significant across time points $\left(F[2,111]=20.1 ; P<.01 ;\right.$ partial eta $\left.^{2}=0.3\right)$. High and moderate pain scores decreased significantly from T0 to T2, and low scores increased (all $P<.01)$; all 3 of these were clinically significant. High scores attributed for $31.6 \%$ of the sample, moderate for $35.1 \%$, and low for $33.3 \%$.

Fatigue: The linear trend was significant across time points, $\left(\mathrm{F}[2,111]=21.9 ; \mathrm{P}<.01 ;\right.$ partial eta $\left.{ }^{2}=0.3\right)$. Low scores significantly increased and high scores significantly decreased (both $P<.01$ ); these were both clinically significant. Moderate scores did not reach either significant change. High scores attributed for $33.3 \%$ of the sample, moderate for $38.6 \%$, and low for $28.1 \%$.

Depression: The linear trend was significant across time points, $\left(\mathrm{F}[2,111]=31.3 ; \mathrm{P}<.01 ;\right.$ partial eta $^{2}$ $=0.4)$. High scores decreased significantly $(P=.02)$ and low scores increased significantly $(P<.01)$; these were both clinically significant. Moderate scores

\section{Table 2. ESAS Scores Across Time Points and} Significance of Change Versus Discharge (T2)

\begin{tabular}{|c|c|c|c|c|c|c|}
\hline & \multicolumn{2}{|c|}{ Low Group } & \multicolumn{2}{|c|}{ Moderate Group } & \multicolumn{2}{|c|}{ High Group } \\
\hline & Mean & SD & Mean & SD & Mean & SD \\
\hline Pain & \multicolumn{2}{|c|}{$n=38$} & \multicolumn{2}{|c|}{$\mathrm{n}=40$} & \multicolumn{2}{|c|}{$n=36$} \\
\hline TO & 1.7 & $1.1^{\mathrm{a}, \mathrm{b}}$ & 4.9 & $0.7^{\mathrm{a}, \mathrm{b}}$ & 8.1 & $1.0^{\mathrm{a}, \mathrm{b}}$ \\
\hline $\mathrm{T} 1$ & 4.6 & $2.4^{\mathrm{a}, \mathrm{b}}$ & 5.2 & $2.3^{a, b}$ & 7.1 & $2.1^{\mathrm{a}, \mathrm{b}}$ \\
\hline T2 (Ref) & 3.2 & 2.7 & 3.9 & 2.3 & 5.4 & 2.8 \\
\hline Fatigue & \multicolumn{2}{|c|}{$n=32$} & \multicolumn{2}{|c|}{$\mathrm{n}=44$} & \multicolumn{2}{|c|}{$n=38$} \\
\hline TO & 2.1 & $1.1^{\mathrm{a}, \mathrm{b}}$ & 5.1 & 0.8 & 8.1 & $0.8^{\mathrm{a}, \mathrm{b}}$ \\
\hline $\mathrm{T} 1$ & 3.9 & 2.3 & 5.6 & 1.8 & 6.6 & $2.1^{\mathrm{b}}$ \\
\hline T2 (Ref) & 3.8 & 2.3 & 5.5 & 2.5 & 5.6 & 2.8 \\
\hline Depression & \multicolumn{2}{|c|}{$\mathrm{n}=77$} & \multicolumn{2}{|c|}{$\mathrm{n}=18$} & \multicolumn{2}{|c|}{$n=19$} \\
\hline TO & 0.8 & $1.1^{\mathrm{a}, \mathrm{b}}$ & 5.0 & 0.8 & 7.8 & $1.1^{\mathrm{a}, \mathrm{b}}$ \\
\hline $\mathrm{T} 1$ & 1.9 & 2.4 & 4.6 & 2.2 & 6.8 & $2.0^{\mathrm{a}, \mathrm{b}}$ \\
\hline T2 (Ref) & 2.2 & 2.4 & 4.6 & 2.7 & 4.3 & 3.2 \\
\hline Anxiety & \multicolumn{2}{|c|}{$n=64$} & \multicolumn{2}{|c|}{$n=30$} & \multicolumn{2}{|c|}{$\mathrm{n}=20$} \\
\hline T0 & 1.0 & $1.2^{\mathrm{a}, \mathrm{b}}$ & 4.8 & $0.7^{\mathrm{a}, \mathrm{b}}$ & 8.2 & $1.1^{\mathrm{a}, \mathrm{b}}$ \\
\hline $\mathrm{T} 1$ & 1.6 & 2.0 & 4.1 & 2.3 & 7.4 & $2.4^{\mathrm{a}, \mathrm{b}}$ \\
\hline T2 (Ref) & 1.9 & 2.3 & 3.5 & 2.6 & 5.1 & 3.8 \\
\hline Well-being & \multicolumn{2}{|c|}{$\mathrm{n}=34$} & \multicolumn{2}{|c|}{$n=48$} & \multicolumn{2}{|c|}{$\mathrm{n}=32$} \\
\hline TO & 1.5 & $1.1^{\mathrm{a}, \mathrm{b}}$ & 5.0 & 0.7 & 8.0 & $1.1^{\mathrm{a}, \mathrm{b}}$ \\
\hline $\mathrm{T} 1$ & 3.6 & 2.2 & 5.1 & 2.0 & 6.4 & 2.6 \\
\hline T2 (Ref) & 3.8 & 2.7 & 4.5 & 2.1 & 5.8 & 2.5 \\
\hline
\end{tabular}

Abbreviations: ESAS, Edmonton Symptom Assessment System; T0, referral; T1, initial consult; $\mathrm{T} 2$, discharge.

astatistically significant difference compared with $\mathrm{T} 2(P \leq .01)$.

${ }^{b}$ Clinically significant difference (change of $\geq 0.5$ SDs and \pm 1 unit). did not reach either significant change. High scores attributed for $16.7 \%$ of the sample, moderate for $15.8 \%$, and low for $67.5 \%$.

Anxiety: The linear trend was significant across time points, $\left(F[2,111]=20.7 ; P<.01 ;\right.$ partial eta $\left.{ }^{2}=0.3\right)$. High and moderate scores significantly decreased and low scores increased significantly (each $P<.01$ ). Decreases in the moderate and high scores were clinically significant. High scores attributed for $17.6 \%$ of the sample, moderate for $26.3 \%$, and low for $56.1 \%$.

Well-Being: The linear trend was significant across

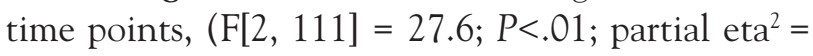
$0.3)$. High scores were significantly lower and low scores were significantly higher (both $P<.01$ ); these were both clinically significant. Moderate scores did not reach either significant change. High scores attributed for $28.1 \%$ of the sample, moderate for $42.1 \%$, and low for $29.8 \%$.
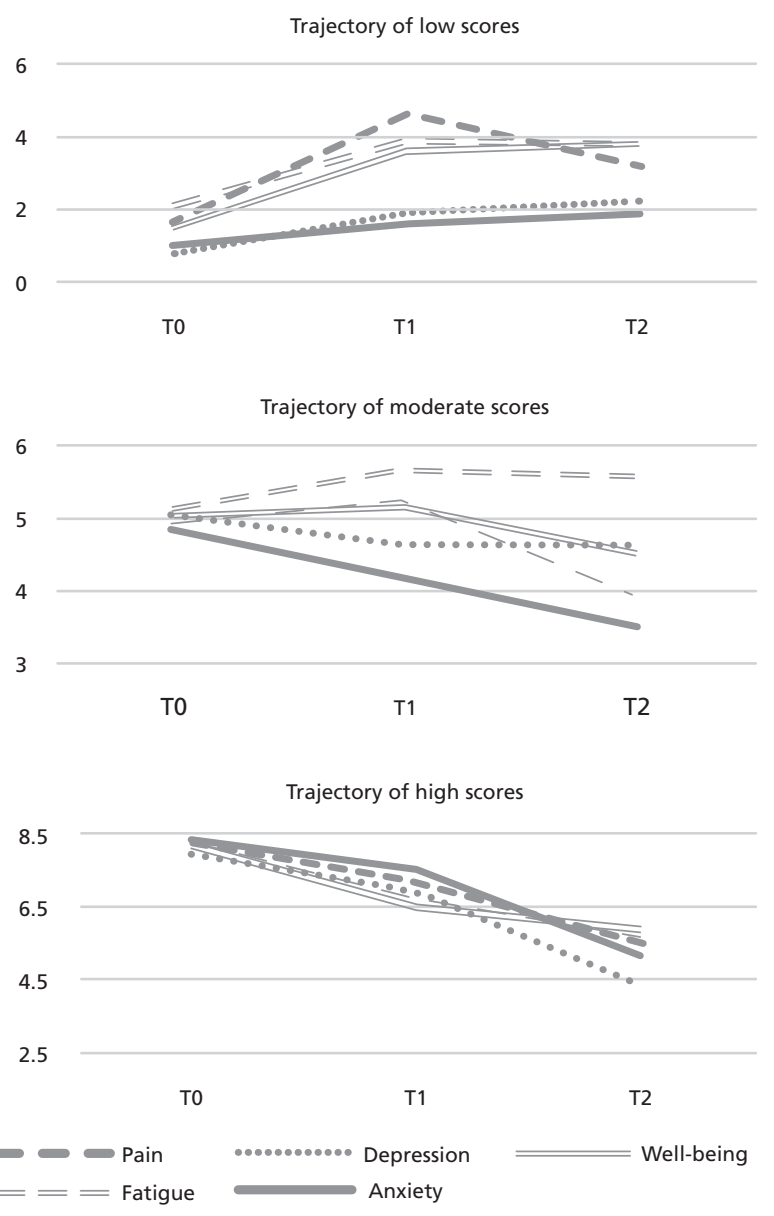

Figure 2. Trajectory of 5 symptoms across referral (T0), initial consult (T1), and discharge (T2). 


\section{Canadian Problem Checklist}

The top 10 endorsed problems included 7 physical and 3 psychosocial items, with sleep (47.4\%), walking/mobility $(42.1 \%)$, and fear $(36.0 \%)$ the most common problems at T1 (Table 3). Cochrane Q analyses of the top 10 revealed significant improvements from $\mathrm{T} 1$ to $\mathrm{T} 2$ in frustration/anger $(P=.02)$, sense of burdening others $(P=.04)$, and sensitivity to cold $(P=.04)$. These analyses were rerun excluding patients who died within 1 month of discharge $(n=100)$, and the same top 10 problems emerged in the same order. However, decreased sleep problems reached significance $(P=.03$; Table 3$)$.

\section{Discussion}

The present study examined patient outcomes for the CCMS. The goal was to examine trends in reported patient outcomes and identify areas of unmet need. This can serve to inform the ongoing service provided and future programming.

A number of interesting trends emerged in our analyses. First, high scores for all 5 symptoms decreased significantly, statistically and clinically, supporting that CCMS intervention may help patients experience relief from their most burdensome symptoms. Pain and anxiety decreased in the moderate ESAS score group, and the low score group reported significant increases in all 5 symptoms. Increasing low scores replicates previous findings in a similar population. ${ }^{22} \mathrm{CPC}$ results revealed improvements in frustration/anger, sense of burdening others, sensitivity to cold, and sleep (when excluding patients within the last month of life), but not in the other 6 endorsed top 10 problems.
Together, these results suggest that this specialized consult service may be most effective for the high-severity symptoms, which were likely the catalyst for referral. This is not necessarily problematic in terms of the CCMS' functioning or approach; it suggests that they are addressing areas of highest need. Discharge interviews may be beneficial to address ongoing symptoms of lesser severity, allowing for further referral or arrangement of resources. The lack of change in 3 of 5 of the moderate symptoms and an increase in all of the low-rated symptoms highlights that ongoing monitoring (ie, $\mathrm{SFD}^{9}$ ) is important to address needs as they arise. ${ }^{10}$ Specialist palliative care specifically for symptom management may not be necessary for these levels of symptom burden, although communication between teams and appropriate follow-up could prevent future symptom flares.

Our findings also reveal that a large number of patients are being referred earlier in the cancer trajectory $(78.8 \%$ and $59.6 \%$ survived $\geq 6$ months in 2015 and 2016, respectively). This is higher than reported referral practices in Canada, with $86.7 \%$ of oncologists referring within the last 6 months of life. ${ }^{23}$ This may suggest a transitioning toward earlier integration of palliative services, which has been found to improve QoL and survival. ${ }^{1,2}$ It may also be influenced by the availability of this team in Calgary compared with other Canadian cities or rural areas. Despite this survival statistic, CCMS referrals are received disproportionately, with certain clinicians or tumor groups referring more often than others. Therefore, it still seems reasonable to hypothesize that patients who could benefit from this service are not referred due to factors such as attitudes or

\begin{tabular}{|c|c|c|c|c|c|c|c|c|}
\hline & & & \multicolumn{3}{|c|}{ Full Sample $(n=114)$} & \multicolumn{3}{|c|}{ Survival $\geq 1$ Month $(n=100)$} \\
\hline & & & $\% \mathrm{~T} 1$ & $\%$ T2 & $P$ Value & $\% \mathrm{~T} 1$ & $\% \mathrm{~T} 2$ & $P$ Value \\
\hline 1 & Physical & Sleep & 47.4 & 35.1 & .06 & 49.0 & 34.0 & $.03 *$ \\
\hline 2 & Physical & Walking/Mobility & 42.1 & 43.0 & 1.00 & 42.0 & 40.0 & .85 \\
\hline 3 & Emotional & Fear & 36.0 & 26.3 & .11 & 35.0 & 23.0 & .06 \\
\hline 4 & Physical & Weight & 33.3 & 25.4 & .22 & 34.0 & 23.0 & .11 \\
\hline 5 & Physical & Concentration/Memory & 29.8 & 28.9 & 1.00 & 34.0 & 29.0 & .42 \\
\hline 6 & Physical & Numbness/Tingling & 29.8 & 28.9 & 1.00 & 31.0 & 27.0 & .56 \\
\hline 7 & Physical & Constipation & 28.1 & 26.3 & .86 & 29.0 & 25.0 & .58 \\
\hline 8 & Emotional & Frustration/Anger & 28.1 & 16.7 & $.02 *$ & 29.0 & 13.0 & $<.01 *$ \\
\hline 9 & Social & Burden to others & 25.4 & 16.7 & $.04 *$ & 25.0 & 14.0 & $<.01 *$ \\
\hline 10 & Physical & Sensitivity to cold & 23.7 & 14.0 & $.04 *$ & 25.0 & 14.0 & $.03^{*}$ \\
\hline
\end{tabular}


Interdisciplinary Palliative Consult

fears about palliative care. ${ }^{23-26}$ Clinicians in oncology need to continue addressing symptom management, performing ongoing systematic screening, and providing appropriate follow-up.

Integration between CCMS and PSRO allows the team to address multifactorial complex suffering (eg, pain, fatigue, and depression ${ }^{6-8}$ ) with different approaches. These complex symptoms can be difficult to treat, yet our results are promising. Through interdisciplinary collaboration, factors influencing a patient's suffering are identified, and treatment planning can ensue with personalized needs in mind. For example, a depression score might be impacted by physical (treatment-related disfigurement ${ }^{27}$ ), biological (inflammation ${ }^{28}$ ), psychological (demoralization, ${ }^{29}$ poor self-esteem ${ }^{27}$ ), and social factors (relationship conflict, ${ }^{27}$ isola$\operatorname{tion}^{28}$ ), or a combination. Team members can easily and quickly make referrals to one another, consult to better understand the patient's overall presentation, and coordinate treatment efforts. Having a psychologist available as a team member helped provide interdisciplinary support and coordinated treatment planning and may have bolstered patient follow-through, perhaps because meeting the psychologist helped familiarize and normalize the process of speaking with a psychologist. Patients may have also felt less invisible or alone in acquiring psychosocial support and were thereby less likely to slip through the cracks.

CCMS has a high attendance rate: 93\% of eligible patients attended T1, and $67.9 \%$ of these completed T2. Only $6.5 \%$ of patients discontinued for unknown reasons, and the remainder received other services or referrals according to need. This suggests that for referred patients, the CCMS model is effective for reaching a population that tends to be overloaded with appointments and experiences multisymptom burden. It does require flexibility on the CCMS's behalf as well as from their TBCC colleagues, who allow consult time within clinics.

An interesting phenomenon that may be manifesting is also a tendency to report more extreme scores when beginning treatment with a new team despite completing SFD measures multiple times before. This resembles regression to the mean, which can theoretically be managed by adding pre-baseline measures. ${ }^{21}$ In our case, having previously completed multiple SFD measures did not appear to mitigate this effect. This may speak to the urgency for timely integration of palliative care, given the potential for abrupt worsening of symptoms, and to the current recommendation in cancer care that SFD measurements be taken minimally at transition points. ${ }^{10}$ Our findings may suggest the importance of taking SFD measurements at more frequent intervals, for the sake of comparing times of transition with times of nontransition. Further investigation of this as a clinical versus statistical trend may be important.

Limitations include that SFD is not diagnostic. It is intended to identify concerns and then address or refer for appropriate support. We were performing a secondary analysis on measures that were gathered for clinical purposes, which may affect responses (eg, Hawthorne effect, or haphazard completion from habituation to completing them at each visit). Dedicated in-person CCMS follow-ups were arranged only for specific issues of concern that could not be addressed by phone. Therefore, many T2 measures were completed during other clinic appointments. A contrast group could have helped us examine these findings further.

A number of implications and future directions are being considered. For patients who present with complex symptomatology, this interdisciplinary approach with biomedical and psychosocial collaboration appears to help attenuate symptom burden. The present analysis has not examined the cumulative effect of the integration; however, this is a traditional approach in palliative care, which aims to treat biopsychosocial-spiritual elements of suffering. Anecdotally, the integration allowed us to reach patients with more longstanding or complex mental health concerns using a coordinated team approach. It will be interesting to examine this in future analyses. With growing incidence of advanced disease and extended survival, an approach such as this for improved QoL will become imperative. The CCMS also offers a novel approach in which we attempt to meet the patients where they are, whether in other clinic appointments or by telephone. This appears to be effective, and presumably makes this service more available to those who live away from major centers. It is a consideration for other care providers and may be interesting to compare empirically to a more traditional center-focused model. Psychosocial follow-ups could have been performed by telephone or telehealth. The difference between in-person and 
Feldstain et al

remote psychosocial sessions may be an interesting area of future evaluation. Finally, the importance of ongoing screening, treatment, and referral becomes evident when we see that even low symptoms fluctuate: symptom burden is not stable nor does it remain at a manageable level over time.

\section{Conclusions}

Our results indicate that a specialized palliative consult service embedded within the cancer center may help patients address symptoms of high severity, including complex symptoms with multiple etiologies. The approach of visiting patients during existing oncology appointments or triaging them to a half-day clinic appears to be beneficial in reaching this pop- ulation of ambulatory patients with high symptom burden. Ongoing considerations include remaining on the radar for patients and professionals to continue addressing palliation needs as they arise, and assuring appropriate referrals to meet patient needs. The interdisciplinary collaboration between CCMS and PSRO allowed for clinicians involved to better understand patient's presenting concerns, address concerns from a number of angles, and facilitate referrals within the team.

\section{Acknowledgments}

The authors wish to thank our Complex Cancer Management Service colleagues, TBCC colleagues, and our patients.

\section{References}

1. Greer JA, Jackson VA, Meier DE, Temel JS. Early integration of palliative care services with standard oncology care for patients with advanced cancer. CA Cancer J Clin CA 2013;63:349-363.

2. Temel JS, Greer JA, Muzikansky A, et al. Early palliative care for patients with metastatic non-small-cell lung cancer. N Engl J Med 2010;363:733742

3. Zimmermann C, Swami N, Krzyzanowska M, et al. Early palliative care for patients with advanced cancer: a cluster-randomised controlled trial. Lancet 2014;383:1721-1730.

4. Saunders C. The evolution of palliative care. Patient Educ Couns 2000;41:7-13.

5. Reeves S, Goldman J, Gilbert J, et al. A scoping review to improve conceptual clarity of interprofessional interventions. J Interprof Care 2011;25:167-174.

6. Berger AM, Mooney K, Banerjee C, et al. NCCN Clinical Practice Guidelines in Oncology. Cancer-Related Fatigue, Version 2.2015. J Natl Compr Canc Netw 2015;13:1012-1039. To view the most recent version of these guidelines, visit NCCN.org.

7. Mehta A, Chan LS. Understanding of the concept of "total pain": a prerequisite for pain control. J Hosp Palliat Nurs 2008;10:26-32.

8. Smith HR. Depression in cancer patients: pathogenesis, implications and treatment. Oncol Lett 2015;9:1509-1514.

9. Bultz BD, Carlson LE. Emotional distress: the sixth vital sign in cancer care. J Clin Oncol 2010;23:6440-641.

10. Cancer Journey Portfolio. Screening for Distress, the 6th Vital Sign: A Guide to Implementing Best Practices in Person-Centred Care. Available at: http:// www.virtualhospice.ca/Assets/Distress-\%20CPAC_20150713161546.pdf. Accessed October 13, 2017

11. Talk About What Matters to You-Putting Patients First. Alberta Health Services Website. Available at: http://www.albertahealthservices.ca/frm20338.pdf. Accessed October 13, 2017.

12. Bruera E, Kuehn N, Miller MJ, et al. The Edmonton Symptom Assessment System (ESAS): a simple method for the assessment of palliative care patients. J Palliat Care 1991;7:6-9.

13. Kirkova J, Davis MP, Walsh D. Cancer symptom assessment instruments: a systematic review. J Clin Oncol 2006;24:1459-1473.

14. Watanabe SM, Nekolaichuk CL, Beaumont C. The Edmonton Symptom Assessment System, a proposed tool for distress screening in cancer patients: development and refinement. Psychooncology 2012;21:977-985.

15. Richardson LA, Jones GW. A review of the reliability and validity of the Edmonton Symptom Assessment System. Curr Oncol 2009;16:55.

16. Graham JW. Missing Data: Analysis and Design. New York, NY: Springer; 2012.

17. Bedard G, Zeng L, Zhang L, et al. Minimal clinically important differences in the Edmonton Symptom Assessment System in patients with advanced cancer. J Pain Symptom Manage 2013;46:192-200.

18. Hui D, Shamieh O, Paiva CE, et al. Minimal clinically important differences in the Edmonton Symptom Assessment Scale in cancer patients: a prospective, multicenter study. Cancer 2015;121:3027-3035.

19. Lynn J, Adamson DM. Living Well at the End of Life: Adapting Health Care to Serious Chronic Illness in Old Age. Available at: https://www.rand. org/content/dam/rand/pubs/white_papers/2005/WP137.pdf. Accessed May 2, 2018.

20. Seow H, Barbera L, Sutradhar R, et al. Trajectory of performance status and symptom scores for patients with cancer during the last six months of life. J Clin Oncol 2011;29:1151-1158.

21. Linden A. Assessing regression to the mean effects in health care initiatives. BMC Med Res Methodol 2013;13:119.

22. Kang JH, Kwon JH, Hui D, et al. Changes in symptom intensity among cancer patients receiving outpatient palliative care. J Pain Symptom Manage 2013;46:652-660.

23. Wentlandt K, Krzyzanowska MK, Swami N, et al. Referral practices of oncologists to specialized palliative care. J Clin Oncol 2012;30:4380-4386.

24. Hui D, Elsayem A, de la Cruz M, et al. Availability and integration of palliative care at US cancer centers. JAMA 2010;303:1054-1061.

25. Lamont EB, Christakis NA. Physician factors in the timing of cancer patient referral to hospice palliative care. Cancer 2002;94:2733-2737.

26. Osta BE, Palmer JL, Paraskevopoulos $T$, et al. Interval between first palliative care consult and death in patients diagnosed with advanced cancer at a comprehensive cancer center. J Palliat Med 2008;11:51-57.

27. Smith JD, Shuman AG, Riba MB. Psychosocial issues in patients with head and neck cancer: an updated review with a focus on clinical interventions. Curr Psychiatry Rep 2017;19:56.

28. Caruso R, Nanni MG, Riba M, et al. Depressive spectrum disorders in cancer: prevalence, risk factors and screening for depression: a critical review. Acta Oncol 2017;56:146-155.

29. Vehling S, Kissane DW, Lo C, et al. The association of demoralization with mental disorders and suicidal ideation in patients with cancer. Cancer 2017;123:3394-3401. 